Check for updates

Cite this: RSC Adv., 2019, 9, 11465

Received 31st December 2018

Accepted 6th April 2019

DOI: $10.1039 / c 8 r a 10663 k$

rsc.li/rsc-advances

\section{Combined molecular docking, homology modelling and density functional theory studies to modify dioxygenase to efficiently degrade aromatic hydrocarbons $\uparrow$}

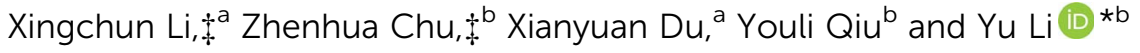

To promote the biodegradation of aromatic hydrocarbons in petroleum-contaminated soils, naphthalene dioxygenase (NDO), which is the key metabolic enzyme that degrades aromatic hydrocarbons, was modified using molecular docking and homology modelling. The novel NDO enzymes screened can efficiently degrade the target aromatic hydrocarbons naphthalene, anthracene, pyrene and benzo[a] pyrene. The docking showed that the key amino acid residues at the binding site of the NDO enzyme include both hydrophilic residues (Asn201, Asp205, His208, His213, His295 and Asn297) and hydrophobic residues (Phe202, Ala206, Val209, Leu307, Phe352 and Trp358), and the hydrophilic residues were replaced by hydrophobic residues to design 54 kinds of NDO enzyme modification schemes. A total of 14 kinds of novel NDO enzymes designed were found to simultaneously increase the binding affinity to the target aromatic hydrocarbons. The energy barrier and rate constant of the degradation reaction for the NDO enzyme modification were calculated using Gaussian09 software and the KiSThelP program. The novel NDO-7 enzyme exhibited decreases in the energy barrier of $76.28,26.35,4.39$ and $1.88 \mathrm{kcal} \mathrm{mol}^{-1}$ and increases in the rate constant of $54,18,12$ and 5 orders of magnitude in the degradation reactions with naphthalene, anthracene, pyrene and benzo[a]pyrene, respectively. These results provide a theoretical basis for the efficient degradation of aromatic hydrocarbons and the modification of their key metabolic enzymes.

\section{Introduction}

The processes of oil exploitation, storage and transportation have released a large amount of hydrocarbons into the soil, resulting in serious environmental pollution. ${ }^{1}$ Oil pollution can destroy the organic composition of the soil, hinder the respiration of plant roots and the absorption of nutrients, and lead to poor plant growth and even death. ${ }^{2}$ Harmful substances in oil enter the human body through the food chain, directly harming human health. Aromatic hydrocarbons are a class of common organic pollutants in petroleum that are difficult to degrade, and some aromatic hydrocarbons have carcinogenic, teratogenic and mutagenic effects. ${ }^{3}$ The microbial degradation method has the advantages of low processing cost, strong repair effects, no secondary pollution, and little impact on the environment. ${ }^{\mathbf{4}, 5}$ It has become an important remediation technology for petroleum-polluted soils, and it is also the primary method

${ }^{a}$ State Key Laboratory of Petroleum Pollution Control, Beijing 102206, China ${ }^{b}$ College of Environmental Science and Engineering, North China Electric Power University, Beijing 102206, China. E-mail: liyuxx8@hotmail.com

$\dagger$ Electronic supplementary information (ESI) available. See DOI: 10.1039/c8ra10663k

\$ These authors contributed equally to this work. of removing aromatic hydrocarbons in the environment. ${ }^{6}$ The reduction or removal of the toxicity of aromatic hydrocarbons by microbial degradation has become a topic of intense research in environmental remediation research.,8

Recently, research on the biodegradation of aromatic hydrocarbons has intensified, including screening for aromatic hydrocarbon-degrading strains, ${ }^{9}$ degradation characteristics, ${ }^{10}$ metabolic pathways, ${ }^{11}$ and degradation mechanisms ${ }^{12}$ and the construction of efficient genetically engineered bacteria. ${ }^{\mathbf{1 3}}$ Studies reported that a variety of microbial strains to degrade different types of aromatic hydrocarbons in petroleum have been screened out. ${ }^{14,15}$ However, some strains barely adapt to the environment and reproduce at a slow rate, so it is difficult to achieve a degradation rate of aromatic hydrocarbons that meets processing requirements. ${ }^{10} \mathrm{Wu}$ et al. showed that the microbial degradation of aromatic hydrocarbons was achieved by a series of enzymatic reactions, ${ }^{\mathbf{1 6}}$ and the degradation rate depended on the activity of microbial metabolic enzymes, particularly key metabolic enzymes. ${ }^{17}$ Studies have shown that the aromatic ring hydroxylation dioxygenase and the aromatic ring cleavage dioxygenase are two key metabolic enzymes in aromatic hydrocarbon degradation, and the aromatic ring hydroxylation dioxygenase controls the addition of oxygen to benzene rings, 
which is the rate-limiting step of the microbial degradation reaction, determining the rate of the entire degradation process of aromatic hydrocarbons. ${ }^{18}$ Naphthalene, anthracene, pyrene and benzo[a]pyrene are model compounds in the study of aromatic hydrocarbon degradation mechanisms, ${ }^{\mathbf{9}, 10}$ and their degradation pathways have been deduced in previous studies. ${ }^{19-21}$ Lee et al. reported that naphthalene was hydroxylated to cis-1,2-dihydroxy-1,2-dihydronaphthalene by the catalysis of dioxygenase and was completely degraded to $\mathrm{CO}_{2}$ and $\mathrm{H}_{2} \mathrm{O}$ by a series of enzymes. ${ }^{22}$ Moody et al. found that anthracene was hydroxylated by dioxygenase during the degradation of anthracene by Mycobacterium sp. PYR-1, which formed cis-1,2dihydroxy-1,2-dihydroanthracene, and was then further metabolized. ${ }^{23}$ Zhong et $a .^{24}$ and Juhasz and Naidu ${ }^{25}$ showed that pyrene and benzo[a]pyrene produced cis-4,5-dihydroxy4,5dihydropyrene and cis-4,5-BaP-dihydrodiol, respectively, under dioxygenase catalysis.

Genetic engineering has been used to screen the genes for enzymes that can degrade aromatic hydrocarbons from the environment with the goal of constructing genetically engineered bacteria and obtaining new degrading enzymes with a broad degradation spectrum and strong degradative ability. However, these genetically engineered bacteria generally have poor genetic stability or biosafety problems due to the difficulty of stably retaining the recombinant plasmids. ${ }^{26}$ The rational design and directed evolution of enzymes make it possible to identify key amino acid residues related to enzyme characteristics based on the structure, function and catalytic mechanism of the enzyme; precisely design mutation sites in the amino acid sequence; and modify specific amino acid residues through substitution, insertion or deletion and other molecular biological methods, thereby modifying the properties of the enzyme. ${ }^{27}$ Zhang et al. studied the effects of amino acid residues at the Cel6A active site of Thermobifida fusca cellulase on its catalytic activity, substrate specificity and ligand affinity, and mutants modified with the amino acid residues Arg237, Glu263, Lys259 and His159 were found to exhibit improved hydrolytic activity towards carboxymethyl cellulose. ${ }^{28}$ Liu et al. used directed evolution to obtain a novel pyrethroid pesticide-degrading enzyme that exhibited significant improvements in thermal stability and enzyme activity, and the degradation rates of cyhalothrin, cypermethrin, fenvalerate and deltamethrin were increased to $92.21 \%, 99.75 \%, 93.21 \%$ and $89.48 \%$, respectively. ${ }^{29}$ However, little research has reported the modification of aromatic hydrocarbon-degrading enzymes using the directed evolution method.

Therefore, we attempted to modify one of the key metabolic enzymes, naphthalene dioxygenase (NDO), which metabolizes the target aromatic hydrocarbons naphthalene, anthracene, pyrene and benzo[a]pyrene, to enhance their microbial degradation rate. First, molecular docking was used to determine the key amino acid residues that allow the NDO enzyme to bind to the target aromatic hydrocarbons. Next, the NDO enzyme was modified using rational design methods, and novel NDO enzyme structures were constructed using the homology modelling method. The binding affinity of the novel NDO enzymes to the target aromatic hydrocarbons was evaluated by molecular docking, and the changes in the energy barrier $(\Delta E)$ and rate constant $(k)$ of the modified NDO enzyme reaction were calculated using Gaussian09 software and the KiSThelP program to identify the dioxygenase that most efficiently metabolized the target aromatic hydrocarbons. This study is expected to provide a theoretical basis for the efficient degradation of aromatic hydrocarbons in oil-contaminated soil, the degradation mechanism of aromatic hydrocarbons and the modification of their key metabolic enzymes.

\section{Materials and methods}

\section{Molecular docking method}

The docking of aromatic hydrocarbons with the NDO enzyme was conducted using the Surflex-Dock module in the SYBYL-X 2.0 software (Tripos Inc., St. Louis, MO). The molecular structures of naphthalene, anthracene, pyrene and benzo[a]pyrene were obtained in the Sketch molecular module. Geometric and energy optimization of these compounds was conducted with MMFF94 charges in the Tripos force field and an energy convergence gradient of $0.001 \mathrm{~kJ} \mathrm{~mol}^{-1}$ and a maximum of 1000 iterations. ${ }^{30}$ The protein structure of the NDO enzyme was obtained from the Protein Data Bank (http://www.rcsb.org/pdb) by the PDB ID 107G. ${ }^{31}$ The National Center for Biotechnology Information (NCBI) provided information about the amino acid residues around the catalytically active region, helping to determine the binding site of the NDO enzyme.

Before molecular docking, the protein receptor was pretreated by adding point charges and polar hydrogen atoms as well as removing water molecules, metal ions and ligands. The parameters of the docking process were set to a threshold of 0.5 , a bloat coefficient of 0 and the default values for the other parameters. ${ }^{32}$ After molecular docking, the interactions between the receptor and the ligand were evaluated by the scoring function factoring in hydrophobicity, polarity, entropy and solvation, which was expressed in units of $-\lg \left(K_{\mathrm{d}}\right)$, where $K_{\mathrm{d}}$ represents the dissociation constant. ${ }^{33,34}$ Generally, a higherscoring function showed stronger binding of the receptor with the ligand.

\section{Homology modelling method}

Homology modelling is a well-recognized method to predict and construct receptor proteins based on similar existing protein structures. ${ }^{35}$ The amino acid residue sequence of the NDO enzyme (PDB ID: 1O7G) was acquired from the NCBI database (https://www.ncbi.nlm.nih.gov/protein/107G_A). The key amino acid residues at the binding site of the NDO enzyme (PDB ID: 1O7G) were substituted through rational design to obtain a new NDO enzyme amino acid residue sequence. The new NDO enzyme amino acid sequences and the template NDO enzyme protein were submitted to the SWISS-MODEL server (http://www.swissmodel.expasy.org) in the Automated Protein Modelling Server. The homology modelling method was used to construct the novel NDO enzyme protein structure. The structural rationality of the novel NDO enzyme proteins constructed was evaluated using the Ramachandran conformational map in 
the online PROCHECK evaluation server (http:// services.mbi.ucla.edu/SAVES/). ${ }^{36,37}$

\section{Gaussian calculation method}

Gaussian09 software was used to optimize the structure of naphthalene, anthracene, pyrene and benzo $[a]$ pyrene molecules at the B3LYP/6-31G* basis set level based on density functional theory (DFT). ${ }^{38}$ The transition state (TS) and energy barrier $(\Delta E)$ of the degradation reaction were calculated at the same base set level. The transition state was required to have only one virtual frequency and the intermediate to have no virtual frequency. The transition state was also verified by the Intrinsic Reaction Coordinate (IRC) ${ }^{39,40}$ Additionally, the KiSThelP programme was used to calculate the rate constant $(k)$ of the target aromatic hydrocarbon degradation reaction before and after the NDO enzyme modification at $298 \mathrm{~K}$ based on the transition state theory. ${ }^{41}$

\section{Results and discussion}

The key metabolic enzyme (NDO enzyme) that degrades the target aromatic hydrocarbons naphthalene, anthracene, pyrene and benzo $[a]$ pyrene was modified in this study. Molecular docking was used to determine the key amino acid residues involved in the binding of the target aromatic hydrocarbons to the NDO enzyme. NDO enzyme modification schemes were constructed, and structures of the novel NDO enzyme proteins were obtained using homology modelling. The highly efficient novel NDO enzymes were further evaluated and screened using Gaussian09 software and the KiSThelP programme.
Docking conformation of the target aromatic hydrocarbons and the NDO enzyme

The aromatic ring-hydroxylating dioxygenase was found to be one of the key metabolic enzymes in the microbial degradative pathways of naphthalene, anthracene, pyrene and benzo $[a]$ pyrene in previous studies (see Fig. 1). It catalyses the hydroxylation of aromatic hydrocarbons and promotes the conversion of aromatic hydrocarbons into cis-dihydrodiol compounds, which is the rate-limiting step of the microbial degradation reactions. ${ }^{22-25}$ Kauppi et al. ${ }^{42}$ reported that the NDO enzyme from Pseudomonas NCIB9816-4 was an aromatic ringhydroxylating dioxygenase and a multi-component enzyme system, including an iron thioproteinase consisting of phnAc and phnAd, a reductase consisting of phnAa and an iron oxidoreductase consisting of phnAb. The reductase releases electrons from $\mathrm{NAD}(\mathrm{P}) \mathrm{H}$ and transfers electrons to the iron oxidoreductase; the iron oxidoreductase transfers electrons to oxygenase; and the oxygenase can catalyse the dihydroxylation of various aromatic hydrocarbons.

In this study, the protein structure of the NDO enzyme (PDB ID: 1O7G) was obtained from the PDB database, and the molecular docking technique was used to simulate the binding conformation of the target aromatic hydrocarbons naphthalene, anthracene, pyrene and benzo[a]pyrene with the NDO enzyme using the Surflex-Dock module. To test the reliability of the Surflex-Dock docking, the natural ligand extracted from the NDO enzyme (PDB ID: 1O7G) was re-docked into the crystal structure of the NDO enzyme. Comparison of the natural ligand docking conformation with the protein/ligand complex crystal structure showed that the root-mean-square deviation (RMSD) for the docking conformations and the heavy (non-hydrogen) atoms in the complex crystal structures was $0.2906 \AA(<2 \AA)$,

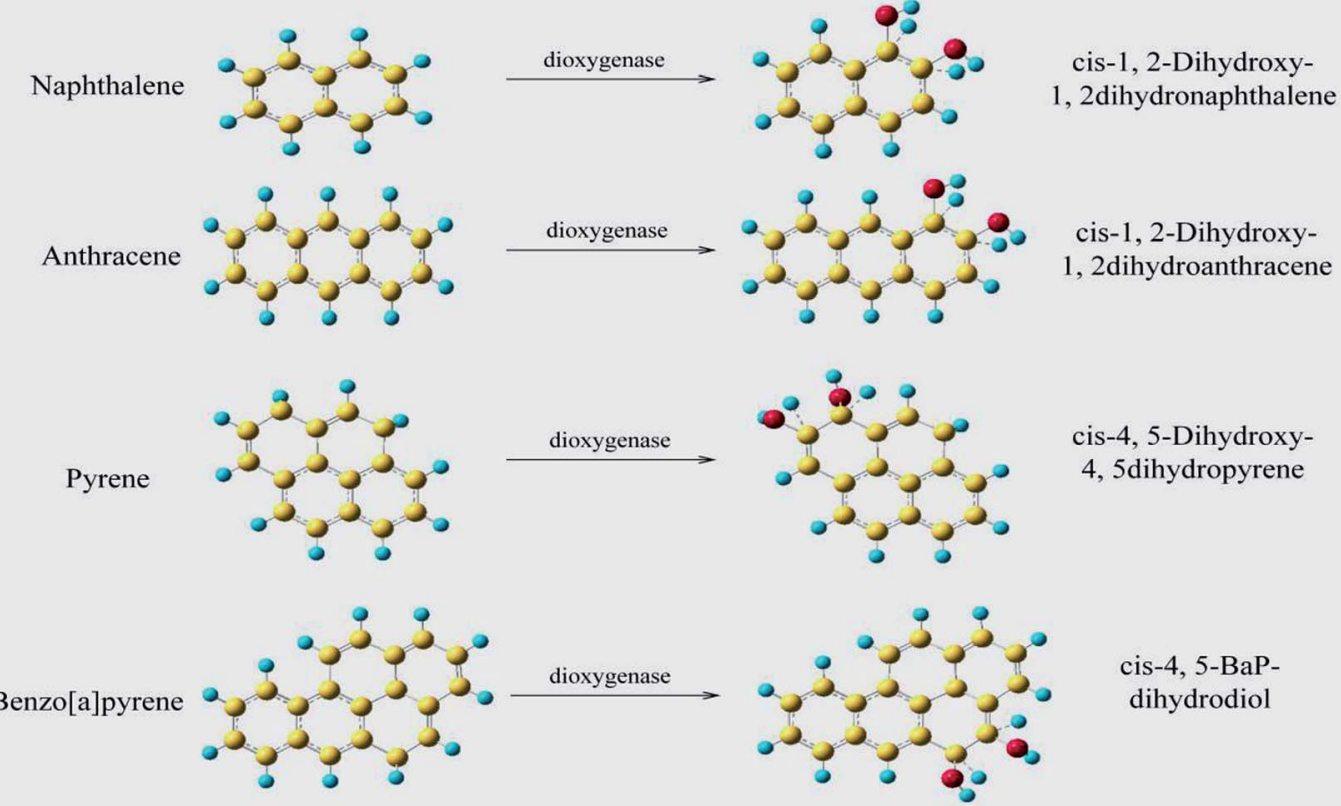

Fig. 1 The rate-limiting step and key metabolic enzyme of the degradation pathway of aromatic hydrocarbons naphthalene, anthracene, pyrene and benzo[a]pyrene. 
indicating the rationality and reliability of the ligand-binding pattern in the molecular docking. ${ }^{43}$

The NCBI database was used to query the structural information of the NDO enzyme (PDB ID: 107G) (https:// www.ncbi.nlm.nih.Gov/protein/107G_A) and obtain the amino acid residues contained in the catalytically active region of the NDO enzyme (PDB ID: 1O7G) to determine the binding site of the target aromatic hydrocarbons to the NDO enzyme (PDB ID: 107G). The target aromatic hydrocarbons naphthalene, anthracene, pyrene and benzo[a]pyrene were docked with the NDO enzyme (PDB ID: 107G) by the Surflex-Dock module of the SYBYL-X2.0 software. As shown in Fig. 2, the docking conformation at the binding site of the target aromatic hydrocarbons naphthalene, anthracene, pyrene and benzo[a]pyrene with the NDO enzyme (PDB ID: 107G) showed that the amino acid residues within a range of $3 \AA$ from the target aromatic hydrocarbons naphthalene, anthracene, pyrene and benzo[ $a]$ pyrene included Asn201, Phe202, Asp205, Ala206, His208, Val209, His213, His295, Asn297, Leu307, Phe352 and Trp358. This list is consistent with the amino acid residues in the catalytically active region of the NDO enzyme (PDB ID: 107G). A compound has been found to interact with a receptor primarily through nonbonding interactions, including hydrophobic and electrostatic interactions, hydrogen bonds and van der Waals forces, and the amino acid residues around the binding site were the primary moieties involved, designated key amino acid residues. ${ }^{44}$ Therefore, it can be preliminarily determined that the amino acid residues described above are the key amino acid residues in which the NDO enzyme (PDB ID: 1O7G) directly interacts with the aromatic hydrocarbons naphthalene, anthracene, pyrene and benzo $[a]$ pyrene.

In further exploration of the interaction between the target aromatic hydrocarbons and the NDO enzyme (PDB ID: 1O7G), the docking conformation in Fig. 2(A) showed that the naphthalene molecule was surrounded by a hydrophobic binding cavity composed of Phe202, Ala206, Val209, Leu307, Phe352 and Trp358 at the binding site of the NDO enzyme (PDB ID: 1O7G). Although there were also hydrophilic amino acid residues Asn201, Asp205, His208, His213, His295 and Asn297 throughout, the hydrophobic naphthalene easily formed a strong hydrophobic interaction with the hydrophobic amino acid residues at the binding site of the NDO enzyme (PDB ID: $107 \mathrm{G})$, which had a stable affinity between the two and thus was conducive to the degradation reaction of naphthalene. Fig. 2(B)-(D) show the docking conformations of the aromatic hydrocarbons anthracene, pyrene and benzo[a]pyrene with the NDO enzyme (PDB ID: 1O7G), respectively. Similarly, the hydrophobic anthracene, pyrene and benzo[a]pyrene formed
(A)

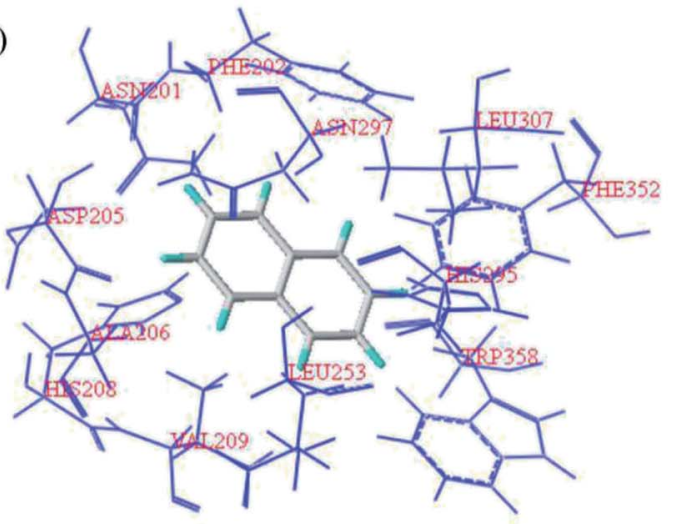

(C)

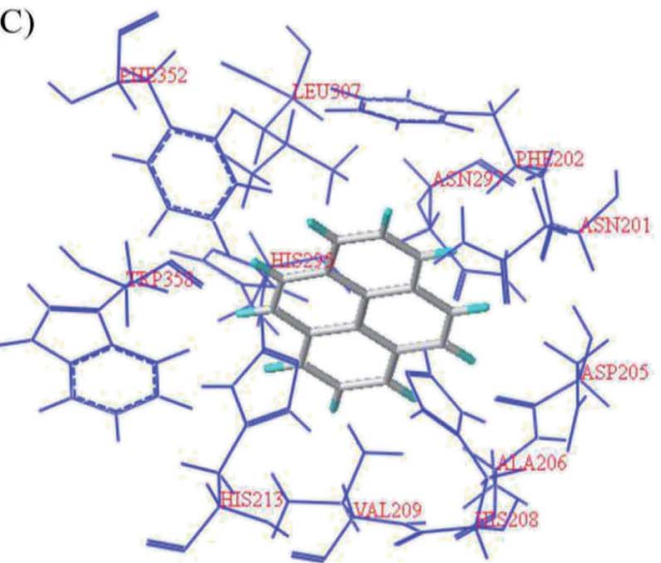

(B)
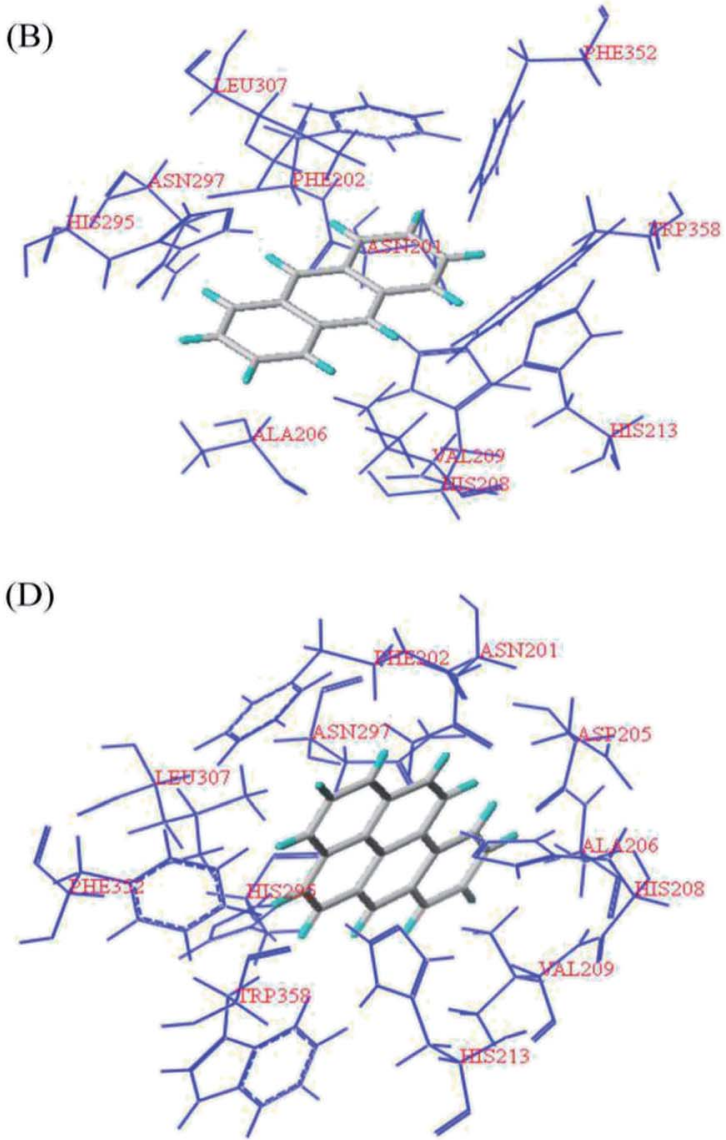

Fig. 2 Docking conformation at the binding site of the target aromatic hydrocarbons and the NDO enzyme (PDB ID: 1O7G); (A) naphthalene; (B) anthracene; (C) pyrene, and (D) benzo[a]pyrene. 
hydrophobic interactions with the hydrophobic amino acid residues Phe202, Ala206, Val209, Leu307, Phe352 and Trp358 at the binding site of the NDO (PDB ID: 1O7G), which facilitated their degradation.

Li et al. explored the interaction between the naphthalenedegrading enzyme cis-1,2-dihydroxy-1,2-dinydronaphthalene dehydrogenase and its substrate cis-1,2-dihydroxy-1,2dinydronaphthalene by molecular docking and found that the increase in the hydrophobicity of the amino acid residues at the binding site could increase their affinity, and stronger binding could further increase the enzymatic reaction rate of naphthalene degradation..$^{45}$ Jin et al. studied the degradation mechanism of the target JPN2-NDO enzyme for pyrene and showed that the hydrophobic interaction played an important role in the binding of pyrene to the target JPN2-NDO enzyme. When the hydrophilic amino acid residue Thr308 of the template 107N enzyme was replaced by the hydrophobic amino acid residue Val232 in the target JPN2-NDO enzyme, the hydrophobic interaction at the binding site was enhanced, which was more favourable for the binding of pyrene. ${ }^{46}$ Therefore, this study attempted to replace the hydrophilic amino acid residues at the binding site of the NDO enzyme (PDB ID: 1O7G) with hydrophobic amino acid residues to increase the affinity between the target aromatic hydrocarbons and the NDO enzyme (PDB ID: $107 \mathrm{G})$, thereby increasing the degradation rate of the target aromatic hydrocarbons naphthalene, anthracene, pyrene and benzo[ $a]$ pyrene by the NDO enzyme.

\section{Modification of the NDO enzyme based on the homology modelling method}

Rational design of an enzyme can identify the key amino acid residues related to enzyme properties based on the structure, function and catalytic mechanism of the enzyme and precisely design mutation sites at which to substitute, insert or delete specific amino acid residues in the amino acid sequence, thereby modifying the properties of the enzyme. Hakamada et al. mutated the Asn179, Asp194 and Glu137 residues of bacterial alkaline cellulase to Lys, and the thermal stability of the enzyme was significantly improved after the modification. ${ }^{47}$ Zhang et al. studied the effects of the amino acid residues at the Cel6A binding site of Thermobifida fusca cellulase on its catalytic activity, substrate specificity and ligand affinity and found that after the modification of the amino acid residues Arg237, Glu263, Lys259 and His159, the mutant had improved hydrolytic activity towards carboxymethyl cellulose. ${ }^{28}$ Based on the hydrophilic or hydrophobic characteristics of 20 amino acid residues (Table A1 $\dagger$ ), the selected hydrophilic amino acid residues Asn201, Asp205, His208, His213, His295 and Asn297 at the binding site of the NDO enzyme (PDB ID: 1O7G) were replaced by the hydrophobic amino acid residues Ile, Phe, Val, Leu, Trp, Met, Ala, Tyr and Pro, respectively, and 54 kinds of modification schemes for individual amino acid residues of the NDO enzyme (PDB ID: 1O7G) were obtained (shown in Table 1).

Homology modelling is a calculation method to establish a three-dimensional molecular structure based on the amino acid sequence of a protein. Research has shown that the higher the identity of the amino acid sequence of the target protein with the template amino acid sequence is, the higher is the accuracy of the established three-dimensional protein structure. $^{36}$ To construct the three-dimensional structure of the modified NDO enzyme, the amino acid sequence of the NDO enzyme (PDB ID: 1O7G) was obtained from the NCBI database (https://www.ncbi.nlm.nih.gov/protein/107G_A). One of the selected hydrophilic amino acid residues, Asn201, Asp205, His208, His213, His295 and Asn297, was replaced with a hydrophobic amino acid residue among Ile, Phe, Val, Leu, Trp, Met, Ala, Tyr and Pro, and a new amino acid sequence was obtained and submitted to the SWISS-MODEL server. The NDO enzyme (PDB ID: 1O7G) with an amino acid sequence similarity of $99.55 \%$ was used as the template protein, and the threedimensional molecular structure of the novel NDO enzymes was constructed using the homology modelling method.

The homology of all the novel NDO enzymes to the template protein was greater than $90 \%$, indicating that the selected template protein was realistic. ${ }^{48}$ The structural rationality of the novel NDO enzyme proteins constructed were evaluated using the Ramachandran conformational map; all of the amino acid residues of the protein were in the allowable region, and the sum of the percentages of residues in the most favoured regions, additional allowed regions and generously allowed regions was greater than $95 \%$, indicating that the structures satisfied the model reliability requirements. ${ }^{36,37}$ As shown in Fig. 3 and 4, using the novel NDO-1 enzyme as an example, the Ramachandran diagram of the protein structure indicated that $90.0 \%$ of the residues were in the most favoured regions; $9.7 \%$ of the residues were in the additional allowed regions, and only $0.3 \%$ of residues were in the disallowed regions, but these residues were not in the active pocket of the NDO-1 enzyme. These analyses indicated that the three-dimensional structure of the NDO-1 enzyme constructed using homology modelling was realistic and reliable. Similarly, the Ramachandran conformational maps of the other 53 novel NDO enzymes were also analysed. The results confirmed that all the novel NDO enzyme protein structures satisfied the rationality requirement that the sum of the percentages of residues in the most favoured regions, additional allowed regions and generously allowed regions was greater than $95 \%$.

\section{Evaluation of the binding affinity of the novel NDO enzymes to target aromatic hydrocarbons}

Studies have shown that increasing the binding affinity of the NDO enzyme to the target aromatic hydrocarbons enhanced the degradative reaction of the NDO enzyme with the target aromatic hydrocarbons. ${ }^{45,49}$ Therefore, to compare the change in the binding affinity between the NDO enzyme and the target aromatic hydrocarbons after the modification, the target aromatic hydrocarbons naphthalene, anthracene, pyrene and benzo[ $a]$ pyrene were molecularly docked with the 54 kinds of novel NDO enzymes using the Surflex-Dock module of the SYBYL-X2.0 software. The Surflex-Dock module derives the scoring function by factoring in the polar interaction, hydrophobic interaction, entropy and solvation between the ligand 
Table 1 Modification schemes of the NDO enzyme (PDB ID: 1O7G) and scoring functions of the novel NDO enzymes docking with the target aromatic hydrocarbons $^{a}$

\begin{tabular}{|c|c|c|c|c|c|c|c|c|c|c|}
\hline \multirow[b]{2}{*}{$\begin{array}{l}\text { Novel NDO } \\
\text { enzymes }\end{array}$} & \multirow{2}{*}{$\begin{array}{l}\text { Original } \\
\text { amino } \\
\text { acid } \\
\text { residues }\end{array}$} & \multirow{2}{*}{$\begin{array}{l}\text { Substituted } \\
\text { amino } \\
\text { acid residues }\end{array}$} & \multicolumn{2}{|c|}{ Naphthalene } & \multicolumn{2}{|c|}{ Anthracene } & \multicolumn{2}{|l|}{ Pyrene } & \multicolumn{2}{|c|}{ Benzo $[a]$ pyrene } \\
\hline & & & $\begin{array}{l}\text { Scoring } \\
\text { function }\end{array}$ & $\begin{array}{l}\text { Change } \\
\text { rate }(\%)\end{array}$ & $\begin{array}{l}\text { Scoring } \\
\text { function }\end{array}$ & $\begin{array}{l}\text { Change } \\
\text { rate }(\%)\end{array}$ & $\begin{array}{l}\text { Scoring } \\
\text { function }\end{array}$ & $\begin{array}{l}\text { Change } \\
\text { rate (\%) }\end{array}$ & $\begin{array}{l}\text { Scoring } \\
\text { function }\end{array}$ & $\begin{array}{l}\text { Change } \\
\text { rate }(\%)\end{array}$ \\
\hline NDO & & & 3.37 & & 4.64 & & 4.95 & & 4.38 & \\
\hline NDO-1 & Asp205 & Ile & 4.23 & 25.56 & 5.02 & 8.11 & 5.66 & 14.37 & 5.15 & 17.75 \\
\hline NDO-2 & & Phe & 3.90 & 15.69 & 4.63 & -0.32 & 5.72 & 15.50 & 5.31 & 21.36 \\
\hline NDO-3 & & Val & 3.74 & 10.83 & 4.81 & 3.61 & 5.74 & 15.90 & 5.84 & 33.39 \\
\hline NDO-4 & & Leu & 3.61 & 7.13 & 4.91 & 5.71 & 5.79 & 16.96 & 5.87 & 34.10 \\
\hline NDO-5 & & $\operatorname{Trp}$ & 3.94 & 16.98 & 4.92 & 6.00 & 5.85 & 18.25 & 5.31 & 21.37 \\
\hline NDO-6 & & Met & 3.67 & 8.87 & 4.91 & 5.88 & 5.78 & 16.67 & 5.60 & 27.87 \\
\hline NDO-7 & & Ala & 3.99 & 18.30 & 4.91 & 5.86 & 5.81 & 17.28 & 5.93 & 35.56 \\
\hline NDO-8 & & Tyr & 4.03 & 19.56 & 4.13 & -10.94 & 5.55 & 12.15 & 5.54 & 26.70 \\
\hline NDO-9 & & Pro & 3.02 & -10.46 & 3.30 & -28.95 & 3.94 & -20.33 & 3.51 & -19.78 \\
\hline NDO-10 & Asn201 & Ile & 3.26 & -3.27 & 4.08 & -12.04 & 4.92 & -0.67 & 5.22 & 19.28 \\
\hline NDO-11 & & Phe & 3.45 & 2.31 & 4.61 & -0.59 & 5.20 & 5.13 & 6.26 & 43.15 \\
\hline NDO-12 & & Val & 3.03 & -10.20 & 4.21 & -9.34 & 4.92 & -0.70 & 5.13 & 17.33 \\
\hline NDO-13 & & Leu & 3.40 & 0.97 & 4.65 & 0.17 & 5.10 & 3.10 & 6.40 & 46.31 \\
\hline NDO-14 & & Trp & 3.34 & -1.01 & 4.28 & -7.73 & 5.02 & 1.42 & 5.50 & 25.75 \\
\hline NDO-15 & & Met & 3.69 & 9.49 & 4.52 & -2.60 & 5.24 & 5.90 & 5.43 & 24.06 \\
\hline NDO-16 & & Ala & 3.35 & -0.63 & 4.26 & -8.28 & 4.97 & 0.34 & 6.19 & 41.53 \\
\hline NDO-17 & & Tyr & 3.39 & 0.45 & 4.66 & 0.39 & 5.16 & 4.23 & 6.15 & 40.52 \\
\hline NDO-18 & & Pro & 3.37 & 0.01 & 4.66 & 0.43 & 4.96 & 0.11 & 6.57 & 50.23 \\
\hline NDO-19 & Asn297 & Ile & 2.52 & -25.23 & 1.99 & -57.16 & 3.11 & -37.19 & 2.96 & -32.24 \\
\hline NDO-20 & & Phe & 2.50 & -25.71 & 1.81 & -60.94 & 3.10 & -37.43 & 2.87 & -34.35 \\
\hline NDO-21 & & Val & 3.47 & 2.87 & 4.31 & -7.12 & 3.53 & -28.68 & 5.16 & 17.98 \\
\hline NDO-22 & & Leu & 2.95 & -12.47 & 4.09 & -11.80 & 1.72 & -65.16 & 2.27 & -48.11 \\
\hline NDO-23 & & Trp & 2.53 & -24.88 & 1.78 & -61.57 & 3.13 & -36.85 & 2.04 & -53.43 \\
\hline NDO-24 & & Met & 2.42 & -28.16 & 1.22 & -73.66 & 2.20 & -55.54 & 1.33 & -69.64 \\
\hline NDO-25 & & Ala & 3.88 & 15.23 & 5.07 & 9.28 & 4.94 & -0.27 & 5.11 & 16.87 \\
\hline NDO-26 & & Tyr & 2.11 & -37.52 & 1.62 & -65.19 & 2.97 & -40.07 & 2.07 & -52.81 \\
\hline NDO-27 & & Pro & 3.59 & 6.40 & 5.12 & 10.23 & 4.84 & -2.17 & 5.35 & 22.23 \\
\hline NDO-28 & His208 & Ile & 3.37 & -0.11 & 4.58 & -1.36 & 5.59 & 12.89 & 4.65 & 6.17 \\
\hline NDO-29 & & Phe & 3.47 & 2.97 & 4.52 & -2.66 & 5.61 & 13.24 & 4.01 & -8.34 \\
\hline NDO-30 & & Val & 3.42 & 1.56 & 4.67 & 0.64 & 5.54 & 11.88 & 4.91 & 12.20 \\
\hline NDO-31 & & Leu & 3.61 & 7.21 & 4.69 & 1.09 & 5.47 & 10.52 & 5.33 & 21.80 \\
\hline NDO-32 & & $\operatorname{Trp}$ & 3.43 & 1.68 & 4.49 & -3.32 & 5.63 & 13.66 & 5.57 & 27.34 \\
\hline NDO-33 & & Met & 3.44 & 1.98 & 4.68 & 0.81 & 5.61 & 13.38 & 4.84 & 10.66 \\
\hline NDO-34 & & Ala & 3.43 & 1.65 & 4.73 & 1.97 & 5.63 & 13.70 & 4.91 & 12.23 \\
\hline NDO-35 & & Tyr & 3.85 & 14.20 & 4.65 & 0.12 & 5.41 & 9.29 & 6.15 & 40.67 \\
\hline NDO-36 & & Pro & 3.50 & 3.95 & 4.61 & -0.61 & 5.49 & 10.99 & 5.07 & 15.92 \\
\hline NDO-37 & His213 & Ile & 2.38 & -29.47 & 2.97 & -35.93 & 3.14 & -36.55 & 2.27 & -48.04 \\
\hline NDO-38 & & Phe & 2.51 & -25.65 & 1.68 & -63.74 & 1.83 & -63.07 & 2.77 & -36.60 \\
\hline NDO-39 & & Val & 2.35 & -30.16 & 2.50 & -46.09 & 2.62 & -47.13 & 2.21 & -49.45 \\
\hline NDO-40 & & Leu & 2.48 & -26.55 & 2.11 & -54.50 & 2.29 & -53.67 & 3.31 & -24.24 \\
\hline NDO-41 & & Trp & 2.42 & -28.25 & 2.14 & -53.83 & 3.02 & -38.93 & 2.35 & -46.27 \\
\hline NDO-42 & & Met & 2.47 & -26.82 & 2.48 & -46.56 & 1.95 & -60.61 & 2.26 & -48.39 \\
\hline NDO-43 & & Ala & 2.44 & -27.51 & 1.64 & -64.68 & 1.94 & -60.79 & 1.70 & -61.22 \\
\hline NDO-44 & & Tyr & 1.24 & -63.37 & 1.77 & -61.88 & 1.92 & -61.19 & 2.29 & -47.71 \\
\hline NDO-45 & & Pro & 2.53 & -24.99 & 2.12 & -54.35 & 2.38 & -51.85 & 1.87 & -57.21 \\
\hline NDO-46 & His295 & Ile & 4.10 & 21.53 & 4.06 & -12.44 & 4.96 & 0.17 & 5.41 & 23.65 \\
\hline NDO-47 & & Phe & 4.21 & 24.96 & 4.63 & -0.23 & 5.55 & 12.19 & 6.42 & 46.70 \\
\hline NDO-48 & & Val & 3.98 & 18.20 & 4.12 & -11.18 & 4.71 & -4.81 & 5.81 & 32.76 \\
\hline NDO-49 & & Leu & 4.07 & 20.75 & 4.17 & -10.09 & 5.14 & 3.81 & 5.84 & 33.56 \\
\hline NDO-50 & & Trp & 2.42 & -28.28 & 2.44 & -47.33 & 2.25 & -54.46 & 2.70 & -38.22 \\
\hline NDO-51 & & Met & 3.99 & 18.35 & 4.17 & -10.08 & 5.46 & 10.19 & 6.38 & 45.75 \\
\hline NDO-52 & & Ala & 4.07 & 20.82 & 4.30 & -7.28 & 4.73 & -4.51 & 5.05 & 15.40 \\
\hline NDO-53 & & Tyr & 2.51 & -25.57 & 1.86 & -59.86 & 3.01 & -39.19 & 2.38 & -45.54 \\
\hline NDO-54 & & Pro & 3.37 & -0.01 & 4.70 & 1.33 & 4.50 & -9.11 & 4.77 & 8.95 \\
\hline
\end{tabular}

${ }^{a}$ The bold words mean the novel NDO enzymes which have simultaneously improved scoring functions with the target aromatic hydrocarbons naphthalene, anthracene, pyrene and benzo[a]pyrene. 


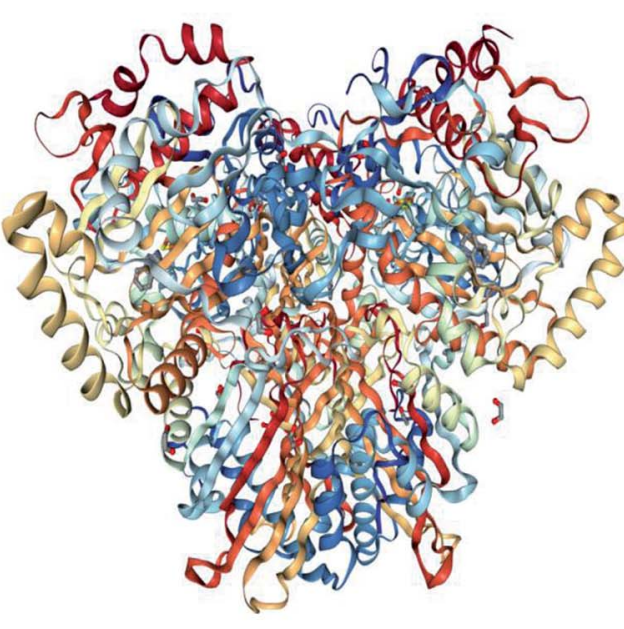

(A)

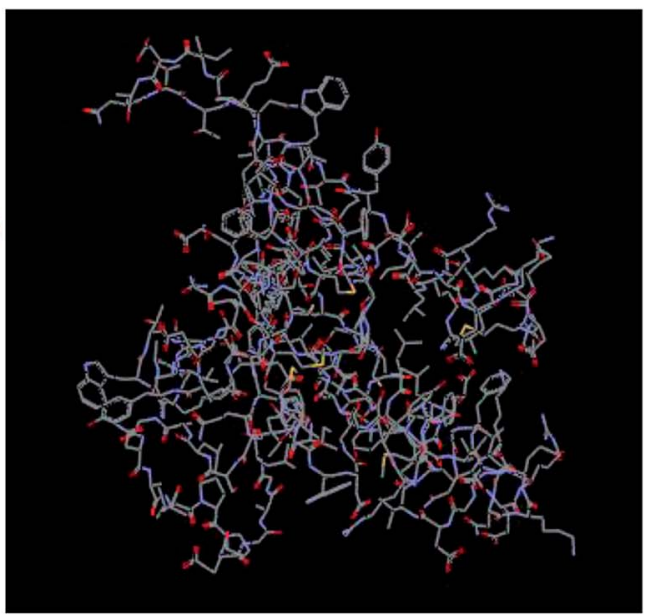

(B)

Fig. 3 Three-dimensional structure of (A) NDO enzyme and (B) novel NDO-1 enzyme.

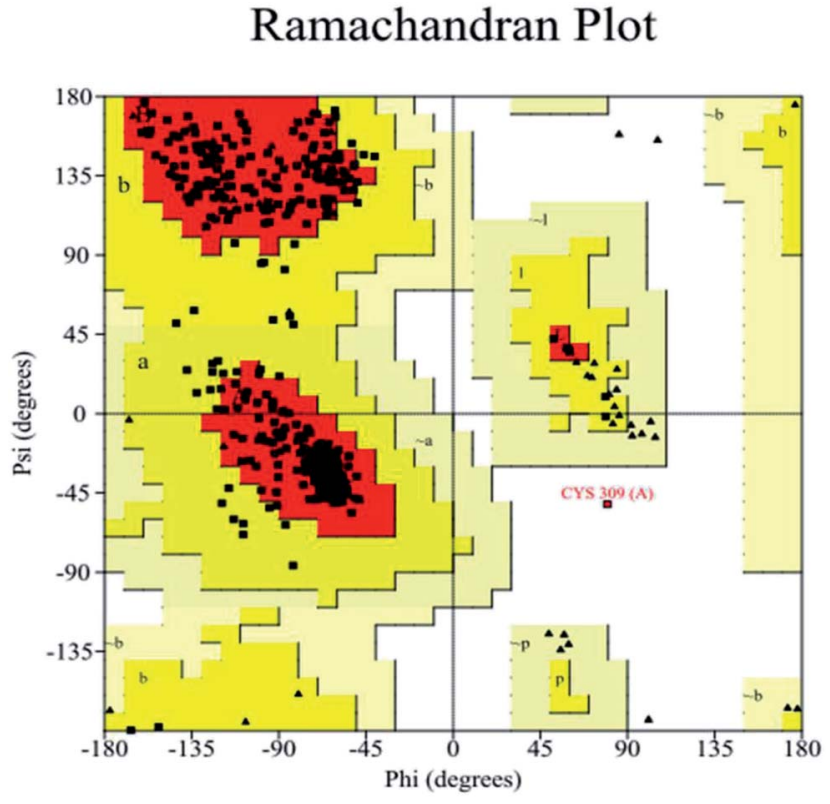

Fig. 4 Ramachandran conformational map of the novel NDO-1 enzyme.

and receptor (Table 1), and a higher scoring function indicates a stronger binding affinity between the ligand and receptor. ${ }^{33,34}$

The scoring functions of the NDO enzyme (PDB ID: 107G) docked with the target aromatic hydrocarbons naphthalene, anthracene, pyrene and benzo[a]pyrene were $3.37,4.64,4.95$ and 4.38, respectively. As shown in Table 1, the scoring functions of the novel NDO enzymes docking with the target aromatic hydrocarbons had different degrees of change compared with the NDO enzyme (PDB ID: 1O7G) before the modification. The novel NDO enzymes with Asn201, Asp205 or His208 at the binding site replaced by hydrophobic amino acid residues had increased or basically unchanged scoring functions with the target aromatic hydrocarbons, while the novel NDO enzymes in which His213, His295 or Asn297 at the binding site replaced had different degrees of decreased scoring functions with the target aromatic hydrocarbons. It can be deduced that Asn201, Asp205 and His208, among the key amino acid residues at the NDO enzyme binding site, played an important role in increasing the binding affinity between the NDO enzyme and the target aromatic hydrocarbons.

In addition, there was some difference in the degree of change in the scoring functions of the novel NDO enzymes docking with the target aromatic hydrocarbons naphthalene, anthracene, pyrene and benzo $[a]$ pyrene. The novel NDO enzymes docking with benzo[ $[a]$ pyrene had scoring functions significantly improved by $6.17-50.23 \%$. The maximum increases in the scoring functions of the novel NDO enzymes docking with the aromatic hydrocarbons naphthalene, anthracene and pyrene were $25.56 \%, 10.23 \%$ and $18.25 \%$, respectively. This may be related to the structures of the target aromatic hydrocarbons naphthalene, anthracene, pyrene and benzo $[a]$ pyrene. It can be established that the 14 kinds of novel NDO enzymes designed have improved scoring functions with the target aromatic hydrocarbons naphthalene, anthracene, pyrene and benzo $[a]$ pyrene, indicating that the NDO enzyme (PDB ID: $107 \mathrm{G})$ modification schemes could enhance the binding affinity between the NDO enzymes and the target aromatic hydrocarbons.

In summary, the 14 kinds of enzyme modification schemes indicated that novel NDO enzymes could be designed by replacing the hydrophilic amino acid residues with hydrophobic amino acid residues at the binding site of the NDO enzyme (PDB ID: 1O7G), which simultaneously increased the binding affinity to the target aromatic hydrocarbons naphthalene, anthracene, pyrene and benzo[a]pyrene and further promoted the degradative reaction of the novel NDO enzymes to the target aromatic hydrocarbons. Among them, the novel NDO enzymes with Asn201, Asp205 and His208 replaced by 
hydrophobic amino acid residues at the binding site showed significantly improved binding affinity to the target aromatic hydrocarbons. In addition, the novel NDO-7 enzyme was found to have the most significant increase in binding affinity with naphthalene, anthracene, pyrene and benzo $[a]$ pyrene, of $18.30 \%, 5.86 \%, 17.28 \%$ and $35.56 \%$, respectively.

\section{Calculation of the energy barrier and rate constant for the degradation reaction of the target aromatic hydrocarbons using the novel NDO enzymes}

As an aromatic ring-hydroxylating dioxygenase, the NDO enzyme can catalyse the hydroxylation of aromatic hydrocarbons and convert them into cis-dihydrodiol compounds, which is the rate-limiting step of the degradation reactions for the target aromatic hydrocarbons naphthalene, anthracene, anthracene and benzo[a]pyrene. ${ }^{22-25}$ To further investigate the effect of the novel NDO enzymes on the degradation of the target aromatic hydrocarbons, the energy barriers and rate constants of the target aromatic hydrocarbons naphthalene, anthracene, pyrene and benzo $[a]$ pyrene degradation by novel NDO enzymes were calculated.

Using the novel enzymes NDO-1, NDO-3, NDO-4, NDO-5, NDO-6 and NDO-7, which had the most significant increased binding affinity to the target aromatic hydrocarbons, the transition state (TS) and energy barrier $(\Delta E)$ of the degradation reaction of the novel NDO enzymes with the target aromatic hydrocarbon naphthalene, anthracene, pyrene and benzo[a] pyrene were calculated using the Gaussian09 software $^{38}$ in which the calculation formula of the energy barrier is shown in eqn (1). In addition, the rate constant $(k)$ of the target aromatic hydrocarbon degradation reaction was calculated using the KiSThelP programme before and after the NDO enzyme modification; ${ }^{41}$ the tunnelling correction was also considered in the Wigner mode, and the resonance frequency correction factor was set to 0.97 . The calculation results are all listed in Table 2 .

$$
\Delta E=E(\mathrm{TS})-\Sigma E(\text { reactant })
$$

Xin et al. calculated the energy barrier of the dioxin-like PCB molecule degradation reaction pathway and found that the lower energy barrier indicated that the degradation reaction occurred more easily. ${ }^{49}$ Huang et al. calculated the relevant reaction energy barrier of the pyrolysis reaction pathways of the cellulose and lignin model syringol and concluded that effectively reducing the energy barrier of the lignin model syringol demethylation reaction was more favourable for the pyrolysis reaction. ${ }^{50}$ As shown in Table 2 , it can be observed that compared with the NDO enzyme (PDB ID: 1O7G), the energy barrier $(\Delta E)$ of the degradation reaction of the novel NDO enzymes and naphthalene, anthracene, pyrene and benzo $[a]$ pyrene decreased. The energy barriers of the naphthalene degradation reaction using the novel NDO-6 enzyme (120.36 kcal $\left.\mathrm{mol}^{-1}\right)$ and novel NDO-7 enzyme (121.28 $\mathrm{kcal} \mathrm{mol}^{-1}$ ) were much smaller than those of the

Table 2 Energy barriers and rate constants for the degradation reaction of the novel NDO enzymes and the target aromatic hydrocarbons

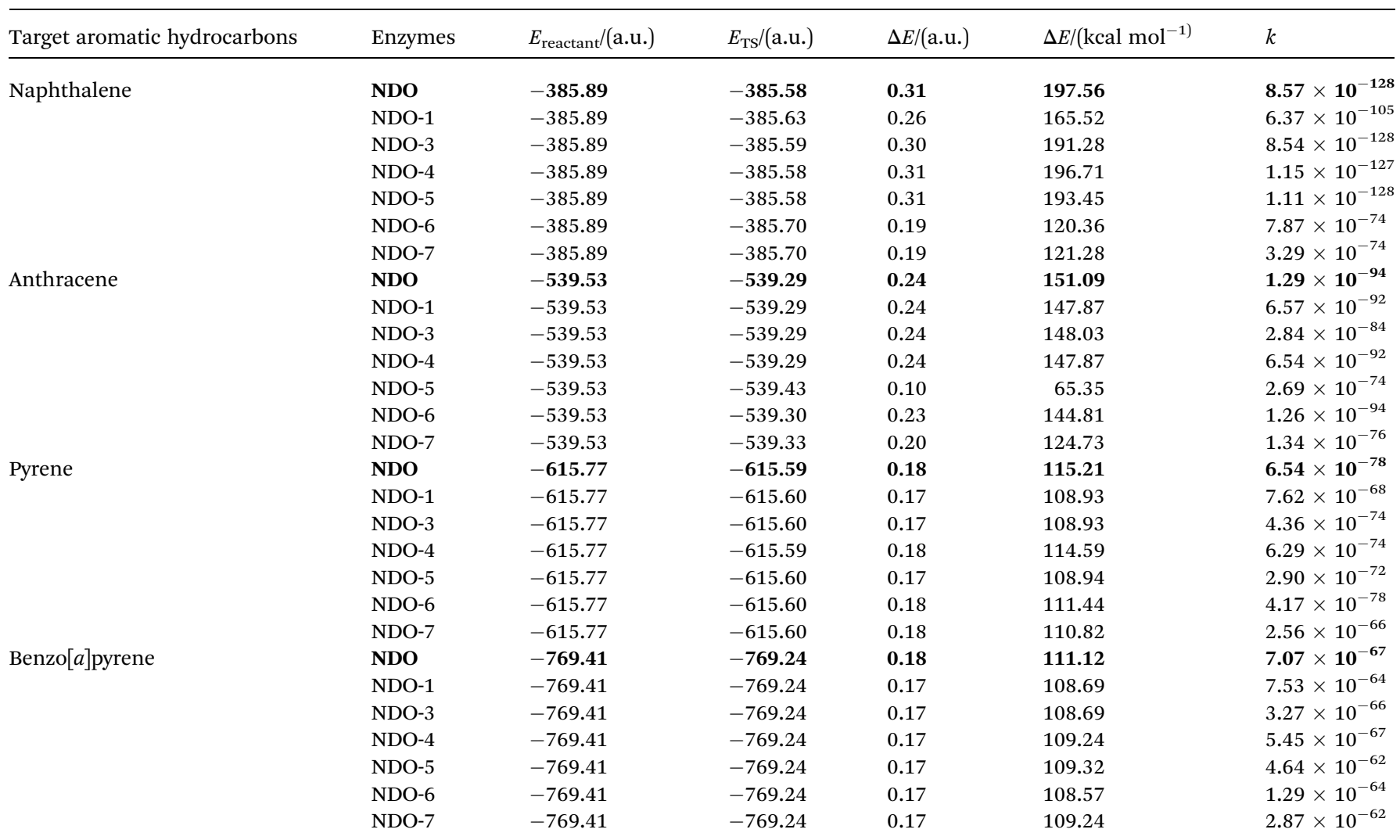


degradation reaction using the NDO enzyme $\left(197.56 \mathrm{kcal} \mathrm{mol}^{-1}\right)$. Compared with the energy barrier of the NDO enzyme ( $\left.151.09 \mathrm{kcal} \mathrm{mol}^{-1}\right)$, the energy barrier of the novel NDO-5 enzyme (65.35 kcal mol ${ }^{-1}$ ) and novel NDO-7 enzyme (124.73 kcal mol ${ }^{-1}$ ) decreased the anthracene degradation reaction by $85.74 \mathrm{kcal} \mathrm{mol}^{-1}$ and $26.36 \mathrm{kcal} \mathrm{mol}^{-1}$, respectively. The energy barriers of the degradation reaction of naphthalene and anthracene using the novel NDO enzymes were significantly reduced. In addition, the novel NDO enzymes also had reduced energy barriers for the degradation of pyrene and benzo[a]pyrene. The novel NDO-1 enzyme had a lower energy barrier $\left(108.93 \mathrm{kcal} \mathrm{mol}^{-1}\right)$ for the pyrene degradation reaction, which was $6.28 \mathrm{kcal} \mathrm{mol}^{-1}$ lower than the NDO enzyme (115.21 $\mathrm{kcal} \mathrm{mol}^{-1}$ ), and the novel NDO-1 enzyme also had a lower energy barrier $\left(108.69 \mathrm{kcal} \mathrm{mol}^{-1}\right)$ for the benzo[a]pyrene degradation reaction with $2.43 \mathrm{kcal} \mathrm{mol}^{-1}$ lower than the NDO enzyme (111.12 $\left.\mathrm{kcal} \mathrm{mol}^{-1}\right)$. In summary, the selected NDO enzyme modification schemes can effectively reduce the energy barrier of the NDO enzyme for the degradative reactions towards naphthalene, anthracene, pyrene and benzo[a]pyrene, resulting in target aromatic hydrocarbons that were more easily degraded by microorganisms.

Abdel-Rahman et al. studied the hydrogen evolution of 2methoxyethanol and investigated the rate constant of the 2methoxyethanol oxidation reaction at different temperatures using the KiSThelP program. ${ }^{51}$ Sun et al. used the KiSThelP program to study the reaction of four carbamate pesticides with $\mathrm{OH}$ free radicals in the atmosphere and calculated the rate constants of a series of reactions based on the typical variational transition state theory and the small curvature tunnelling effect, and the results were consistent with the reaction rate constant measured by the existing experiment. ${ }^{52}$ The calculation results for the rate constants of the target aromatic hydrocarbon degradation reactions by the novel NDO enzymes at $298 \mathrm{~K}$ are shown in Table 2. Compared with the NDO enzyme (PDB ID: 1O7G), the rate constants of the degradation reaction for naphthalene, anthracene, pyrene and benzo $[a]$ pyrene by the novel NDO enzymes have increased by several to tens of orders of magnitude. The rate constants for the naphthalene degradation reaction by the novel NDO-6 and NDO-7 enzymes were $7.87 \times 10^{-74}$ and $3.29 \times 10^{-74}$, respectively, which were significantly higher than that of the NDO enzyme $(8.57 \times$ $10^{-128}$ ) by 54 orders of magnitude. The rate constants of the anthracene degradation reaction by the novel NDO-5 enzyme $\left(2.69 \times 10^{-74}\right)$ and NDO-7 enzyme $\left(1.34 \times 10^{-76}\right)$ significantly increased by 20 and 18 orders of magnitude, respectively, compared with that of the NDO enzyme $\left(1.29 \times 10^{-94}\right)$. The calculated rate constants of pyrene and benzo $[a]$ pyrene degradation by the novel NDO-7 enzyme were $2.87 \times 10^{-62}$ and 2.56 $\times 10^{-66}$, respectively, which were 5 and 12 orders of magnitude higher than that of the NDO enzyme. Thus, the novel NDO enzymes could significantly increase the rate constants of the target aromatic hydrocarbons naphthalene, anthracene, pyrene and benzo $[a]$ pyrene degradation reaction and promote more effective degradation of the target aromatic hydrocarbons by microorganisms.
In summary, the selected novel NDO-1, NDO-3, NDO-4, NDO5, NDO-6 and NDO-7 enzymes could effectively reduce the energy barrier of the degradation reaction of the target aromatic hydrocarbons and significantly increase the rate constant of the degradative reaction, resulting in more rapid degradation of the target aromatic hydrocarbons by microorganisms. Among them, the novel NDO-1, NDO-5 and NDO-7 enzymes had the most significant improvement in the degradation reaction of the target aromatic hydrocarbons. The novel NDO-7 enzyme was found to have energy barriers that decreased by $76.28 \mathrm{kcal} \mathrm{mol}^{-1}, 26.35 \mathrm{kcal} \mathrm{mol}^{-1}, 4.39 \mathrm{kcal} \mathrm{mol}^{-1}$ and $1.88 \mathrm{kcal} \mathrm{mol}^{-1}$ and rate constants that increased by $54,18,12$ and 5 orders of magnitude in the degradation reaction with naphthalene, anthracene, pyrene and benzo[a]pyrene, respectively, compared with those of the NDO enzyme.

\section{Conclusions}

In this paper, the key metabolic enzyme (NDO enzyme) of the aromatic hydrocarbons was docked with naphthalene, anthracene, pyrene and benzo[ $a]$ pyrene. The key amino acid residues at the binding site of the NDO enzyme were determined, including the hydrophilic amino acids Asn201, Asp205, His208, His213, His295 and Asn297 and the hydrophobic amino acids Phe202, Ala206, Val209, Leu307, Phe352 and Trp358. A total of 54 kinds of NDO enzyme modification schemes were designed by substituting the hydrophilic amino acid residues above with hydrophobic amino acid residues, and the novel NDO enzyme protein structures were obtained using the homology modelling method. Among them, 14 kinds of novel NDO enzymes could simultaneously increase the binding affinity (scoring functions) to the target aromatic hydrocarbons. The binding affinity of the novel NDO-7 enzyme to naphthalene, anthracene, pyrene and benzo $[a]$ pyrene increased the most significantly, at $18.30 \%$, $5.86 \%, 17.28 \%$ and $35.56 \%$, respectively. The novel NDO-7 enzyme decreased the energy barriers by $76.28 \mathrm{kcal} \mathrm{mol}^{-1}$, $26.35 \mathrm{kcal} \mathrm{mol}^{-1}, 4.39 \mathrm{kcal} \mathrm{mol}^{-1}$ and $1.88 \mathrm{kcal} \mathrm{mol}^{-1}$ and increased the rate constants by $54,18,12$ and 5 orders of magnitude in the degradation reactions with naphthalene, anthracene, pyrene and benzo[a]pyrene, respectively. These results provide theoretical guidance for the degradation mechanism of aromatic hydrocarbons in petroleum-contaminated soils, the efficient degradation of aromatic hydrocarbons and the modification of their key metabolic enzymes.

\section{Conflicts of interest}

There are no conflicts to declare.

\section{Acknowledgements}

This study was supported by the Open Project Program of State Key Laboratory of Petroleum Pollution Control (No. PPC2016015), CNPC Research Institute of Safety and Environmental Technology. 


\section{References}

$1 \mathrm{M}$. Lin, X. Hu, W. Chen, H. Wang and C. Wang, Int. Biodeterior. Biodegrad., 2014, 94, 176-181.

2 M. R. L. Bonfá, M. J. Grossman, E. Mellado and L. R. Durrant, Chemosphere, 2011, 84, 1671-1676.

3 R. Marcon, G. Bestetti, F. Frati, M. Pepi and F. Baldi, Int. Biodeterior. Biodegrad., 2007, 59, 25-31.

4 S. Kuppusamy, P. Thavamani, M. Megharaj and R. Naidu, Int. Biodeterior. Biodegrad., 2016, 108, 149-157.

5 C. H. Chaîneau, G. Rougeux, C. Yéprémian and J. Oudot, Soil Biol. Biochem., 2005, 37, 1490-1497.

6 C. E. Cerniglia, Bioresour. Technol., 1992, 224, 25-33.

7 S. H. Liu, G. M. Zeng, Q. Y. Niu, Y. Liu, L. Zhou, L. H. Jiang, X. F. Tan, P. Xu, C. Zhang and M. Cheng, Bioresour. Technol., 2017, 224, 25-33.

8 C. Guarino, V. Spada and R. Sciarrillo, Chemosphere, 2017, 170, 10-16.

9 J. S. Seo, Y. S. Keum and Q. X. Li, Int. J. Environ. Res. Public Health, 2009, 6, 278-309.

10 A. K. Haritash and C. P. Kaushik, J. Hazard. Mater., 2009, 169, 1-15.

11 T. M. Martins, O. Núñez, H. Gallart-Ayala, M. C. Leitão, M. T. Galceran and P. C. Silva, J. Hazard. Mater., 2014, 268, 264-272.

12 J. Mao, Y. Luo, Y. Teng and Z. Li, Int. Biodeterior. Biodegrad., 2012, 70, 141-147.

13 G. Minovska, T. Narančić, M. Mandić, L. Šenerović, B. Vasiljević and J. Nikodinović-Runić, Genetika, 2013, 45, 703-716.

14 M. Baboshin, V. Akimov, B. Baskunov, T. L. Born, S. U. Khan and L. Golovleva, Biodegradation, 2008, 19, 567-576.

15 J. Ma, L. Xu and L. Y. Jia, Bioresour. Technol., 2013, 140, 1521.

16 Y. C. Wu, Y. Teng, Z. G. Li and X. W. Liao, Soil Biol. Biochem., 2008, 40, 789-796.

17 M. G. Waigi, F. Kang, C. Goikavi, W. T. Ling and Y. Z. Gao, Int. Biodeterior. Biodegrad., 2015, 104, 333-349.

18 S. Demanèche, C. Meyer, J. Micoud, M. Louwagie, J. C. Willison and Y. Jouanneau, Appl. Environ. Microbiol., 2004, 70, 6714-6725.

19 O. Pinyakong, H. Habe, N. Supaka, P. Pinpanichkarn, K. Juntongjin, T. Yoshida, K. Furihata, H. Nojiri, H. Yamane and T. Omori, FEMS Microbiol. Lett., 2000, 191, 115-121.

20 Y. Prabhu and P. S. Phale, Appl. Microbiol. Biotechnol., 2003, 61, 342-351.

21 S. Mallick, S. Chatterjee and T. K. Dutta, Microbiology, 2007, 153, 2104-2115.

22 H. J. Lee, J. M. Kim, S. H. Lee, M. Park, K. Lee, E. L. Madsen and C. O. Jeon, Microbiology, 2011, 157, 2891-2903.

23 J. D. Moody, J. P. Freeman, D. R. Doerge and C. E. Cerniglia, Appl. Environ. Microbiol., 2001, 67, 1476-1483.

24 Y. Zhong, T. Luan, H. Zhou, C. Lan and N. F. Tam, Environ. Toxicol. Chem., 2006, 25, 2853-2859.
25 A. L. Juhasz and R. Naidu, Int. Biodeterior. Biodegrad., 2000, 45, 57-88.

26 T. M. LaPara, C. H. Nakatsu, L. M. Pantea and J. E. Alleman, Water Res., 2002, 36, 638-646.

27 F. Cheng, L. L. Zhu and U. Schwaneberg, Chem. Commun., 2015, 51, 9760-9772.

28 S. Zhang, B. K. Barr and D. B. Wilson, Eur. J. Biochem., 2010, 267, 244-252.

29 X. L. Liu, Y. H. Liu and X. J. Fan, Acta Sci. Nat. Univ. Sunyatseni, 2017, 56, 111-117.

30 T. A. Halgren, J. Comput. Chem., 1996, 1, 490-519.

31 A. Karlsson, J. V. Parales, R. E. Parales, D. T. Gibson, H. Eklund and S. Ramaswamy, Science, 2003, 299, 10391042.

32 X. Li, L. Ye, X. Wang, X. Wang, H. Liu, Y. Zhu and H. Yu, Toxicol. Appl. Pharmacol., 2012, 265, 300-307.

33 P. A. Holt, J. B. Chairs and J. O. Trent, J. Chem. Inf. Model., 2008, 48, 1602-1615.

34 X. C. Wu, Q. Z. Zhang and J. T. Hu, SAR QSAR Environ. Res., 2016, 27, 147-164.

35 F. Li, X. Li, X. Liu, L. Zhang, L. You, J. Zhao and H. Wu, Environ. Toxicol. Pharmacol., 2011, 32, 478-485.

36 K. Arnold, L. Bordoli, J. Kopp and T. Schwede, Bioinformatics, 2006, 22, 195-201.

37 A. L. Morris, M. W. MacArthur, E. G. Hutchinson and J. M. Thornton, Proteins: Struct., Funct., Genet., 1992, 12, 345-364.

38 M. J. Frisch, G. W. Trucks, H. B. Schlegel, G. E. Scuseria, M. A. Robb, J. R. Cheeseman, G. Scalmani, V. Barone, B. Mennucci, G. A. Petersson, H. Nakatsuji, M. Caricato, X. Li, H. P. Hratchian, A. F. Izmaylov, J. Bloino, G. Zheng, J. L. Sonnenberg, M. Hada, M. Ehara, K. Toyota, R. Fukuda, J. Hasegawa, M. Ishida, T. Nakajima, Y. Honda, O. Kitao, H. Nakai, T. Vreven, J. A. Montgomery, J. J. Peralta, F. Ogliaro, M. Bearpark, J. J. Heyd, E. Brothers, K. N. Kudin, V. N. Staroverov, R. Kobayashi, J. Normand, K. Raghavachari, A. Rendell, J. C. Burant, S. S. Iyengar, J. Tomasi, M. Cossi, N. Rega, J. M. Millam, M. Klene, J. E. Knox, J. B. Cross, V. Bakken, C. Adamo, J. Jaramillo, R. Gomperts, R. E. Stratmann, O. Yazyev, A. J. Austin, R. Cammi, C. Pomelli, J. W. Ochterski, R. L. Martin, K. Morokuma, V. G. Zakrzewski, G. A. Voth, P. Salvador, J. J. Dannenberg, S. Dapprich, A. D. Daniels, Ö. Farkas, J. B. Foresman, J. V. Ortiz and J. F. D. Cioslowski, Gaussian 09, Revision E.01, Gaussian, Inc., Wallingford CT, 2009.

39 R. J. Qu, H. X. Liu, M. B. Feng, X. Yang and Z. Y. Wang, J. Chem. Eng. Data, 2012, 57, 2442-2455.

40 J. Q. Shi, R. J. Qu, M. B. Feng, X. H. Wang, L. S. Wang, S. G. Yang and Z. Y. Wang, Environ. Sci. Technol., 2015, 49, 4209-4217.

41 S. Canneaux, F. Bohr and E. Henon, J. Comput. Chem., 2014, 35, 82-93.

42 B. Kauppi, K. Lee, E. Carredano, R. E. Parales, D. T. Gibson, H. Eklund and S. Ramaswamy, Structure, 1998, 6, 571-586.

43 B. Kramer, M. Rarey and T. Lengauer, Proteins: Struct., Funct., Genet., 1999, 37, 228-241. 
44 W. H. Yang, Z. Y. Wang, H. L. Liu and H. X. Yu, SAR QSAR Environ. Res., 2010, 21, 351-367.

45 X. C. Li, X. Y. Du, Z. H. Chu, J. W. Yang and W. W. Gu, Environ. Chem., 2018, 37, 731-739.

46 J. N. Jin, J. Yao, Q. Y. Zhang and J. L. Liu, Chemosphere, 2016, 164, 379-386.

47 Y. Hakamada, Y. Hatada, T. Ozawa, K. Ozaki, T. Kobayashi and S. Ito, FEMS Microbiol. Lett., 2001, 195, 67-72.

48 P. Benkert, M. Biasini and T. Schwede, Bioinformatics, 2011, 27, 343-350.
49 M. L. Xin, Z. H. Chu and Y. Li, Chem. J. Chin. Univ., 2018, 39, 299-309.

50 J. B. Huang, C. Liu, H. Tong, W. M. Li and D. Wu, Comput. Theor. Chem., 2012, 1001, 44-50.

51 M. A. Abdel-Rahman, S. Hamdy and A. M. El-Nahas, Int. J. Adv. Sci. Tech. Res., 2017, 7, 203-213.

52 S. Sun, K. Zhang and H. Zhang, Theor. Chem. Acc., 2015, 134, 25-39. 